\title{
Nogo-B Facilitates LPS-Mediated Immune Responses by Up-Regulation of TLR4- Signaling in Macrophage RAW264.7
}

\author{
Ying Zhu ${ }^{\mathrm{a}}$ Qiang Tong ${ }^{\mathrm{b}, \mathrm{c}}$ Jia Ye ${ }^{\mathrm{d}}$ Yunye Ning ${ }^{\mathrm{e}}$ Ye Xiong ${ }^{\mathrm{e}}$ Meng Yang Hua Xiao $^{\mathrm{e}}$ \\ Jian $\mathrm{Lu}^{f}$ Jiandong $\mathrm{Li}^{\mathrm{a}}$ Wujian Xug Qiang Li ${ }^{\mathrm{h}}$ \\ aDepartment of Respiratory Medicine, Beijing Army General Hospital, Beijing, 'bepartment of \\ Rheumatology \& Immunology, Shanghai Jiao Tong University Affiliated Sixth People's Hospital, \\ Shanghai, China; 'Department of Applied Sciences-General, University of Huddersfield, Queensgate, \\ Huddersfield, U.K; ${ }^{d}$ Department of Respiratory and Critical Care Medicine, Fuzhou General Hospital \\ of Nanjing Command, Fuzhou, eDepartment of Respiratory and Critical Care Medicine, Changhai

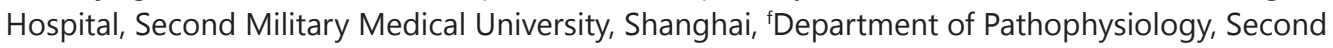 \\ Military Medical University, Shanghai, ${ }^{9}$ Department of Respiratory Medicine, Jinling Hospital, Nanjing, \\ hDepartment of Respiratory Medicine, Shanghai Jiao Tong University Affiliated First People's Hospital, \\ Shanghai, China
}

\section{Key Words}

Macrophage $\cdot$ Nogo-B $\cdot \mathrm{MCP}-1 \cdot$ Immune response $\cdot$ MAP kinase

\begin{abstract}
Background/Aims: Nogo- $B$, a member of the reticulon family of proteins, is mainly located in the endoplasmic reticulum (ER). Here, we investigate the function and mechanism of Nogo- $B$ in the regulation of TLR4-associated immune responses in the macrophage cell line of RAW264.7. Methods: Nogo-B was up- and down-regulated through the use of appropriate adenoviral vectors or siRNA, and the effects of Nogo-B on macrophages under liposaccharide (LPS) stimulation were evaluated via western blotting, immunofluorescence, enzyme-linked immunosorbent assay (ELISA), flow cytometric analysis, and transwell assay. Results: Our data indicates that the protein of Nogo-B was down-regulated in a time- and dose-dependent manner following LPS administration in the macrophage. Nogo-B overexpression increased the production of inflammatory cytokines (MCP-1, TNF- $\alpha$, IL-1 $\beta$, and TGF- $\beta$ ), enhanced macrophage migration activities, activated major histocompatibility complex II (MHC II), and elevated the expression of macrophage scavenger receptor 1(MSR1), all of which suggest that Nogo-B is necessary for immune responses and plays an important role in regulating macrophage recruitment. Mechanistically, Nogo-B may enhance TLR4 expression in macrophage surfaces, activate mitogen-activated protein kinase (MAPK) pathways, and initiate inflammatory responses. Conclusion: These findings illustrate the key regulatory functions of Nogo-B in facilitating LPS-mediated immune responses through promoting the phosphorylation of MAP kinase.

Y. Zhu, Q. Tong and J. Ye contributed equally to this article.




\section{Introduction}

Toll-like receptors (TLRs) are pattern-recognition receptors which play critical roles in triggering innate responses and regulating adaptive immunity [1]. Thirteen TLRs have been found to specifically recognize different pathogen-associated molecular patterns (PAMPs) derived from invading microbes, such as cell surface lipids or proteins, and nucleic acids within the cytosolic endosomal compartments [2, 3]. In particular, the TLR4 receptor is well known to recognize liposaccharide (LPS), a common immunostimulatory bacterial membrane component located in the outer membrane of Gram-negative bacteria [4-6]. Transcription factor nuclear factor- $\kappa \mathrm{B}(\mathrm{NF}-\kappa \mathrm{B})$ is the most important signaling molecule induced by TLRs, as it elicits the expression of immune and inflammatory genes, causing the secretion of cytokines and chemokines such as tumor necrosis factor- $\alpha$ (TNF- $\alpha$ ), interleukin$1 \beta(\mathrm{IL}-1 \beta)$, and monocyte chemoattractant protein-1 (MCP-1) to regulate innate and acquired immune responses $[7,8]$. To date, various signaling molecules or proteins have been shown to regulate TLR pathways to prompt immunological homeostasis, and screening of these molecules is an effective approach to modulating innate immune responses.

Nogo-B, also known as RTN4-B1, ASY, RTNXs, and Foocen-M, is a reticulon superfamily protein located predominantly in the endoplasmic reticulum (ER) retrieval motif and less so in the cell surface[9]. Nogo has three isoforms: A, B, and C[10]. Nogo-A is the largest variant, mainly present in white-matter oligodendrocytes and identified to be a neuronal regeneration inhibitor, playing a variety of roles in neurodegenerative diseases. Nogo-C has been found to be expressed at high levels in skeleton muscles, but its functions are poorly documented [11]. As a splice variant of Nogo-A, Nogo-B is expressed ubiquitously in peripheral tissues $[12,13]$ and is involved in apoptosis regulation, vasculature remodeling, axonal regeneration, wound healing, and tissue repair as well as inflammatory processes [14-16].

Recently, studies on Nogo-B have focused on its general role in regulating macrophage responses, including increasing chemokine-mediated monocyte/macrophage migration and infiltration, reducing the extent of ischemic injuries, and promoting wound healing [17, 18]. Marin et al. have demonstrated a dramatic delay in the recruitment of macrophages in Nogo-B-disrupted mice in vivo, consistent with the findings of Schanda et al. that Nogo-B deficiency is associated with reduced promotion of macrophage homing and functional recovery $[17,19]$. Thus, it has been suggested that endogenous Nogo-B may be implicated in the control of macrophage activities and trafficking [20]. However, whether endogenous Nogo-B is associated with the regulation of macrophages in immune responses aroused by LPS is unclear.

In this study, we aim to further explore the role of Nogo-B in manipulating macrophage activity and uncover its possible mechanism. We have found an activated LPS-mediated immune response by Nogo-B via the TLR4/MAPK pathway, and our study therefore reveals a novel connection between Nogo-B modulation and immune response in macrophages.

\section{Materials and Methods}

\section{Cell preparation, culture and transfection}

The mouse macrophage cell line RAW264.7 was obtained from American Type Culture Collection and cultured in a Dulbecco modified Eagle medium (DMEM) (Gibco) in 10\% fetal bovine serum (FBS) (Gibco) and a penicillin-streptomycin solution (100 U/mL penicillin, $100 \mathrm{ng} / \mathrm{mL}$ streptomycin) (Hyclone) in a humidified incubator containing $5 \% \mathrm{CO}_{2}$ at $37{ }^{\circ} \mathrm{C}$. Macrophage RAW264.7 cells were seeded in a 24 -well plate at a density of $1 \times 10^{5}$ cells per well and transfected with an INTERFERin SiRNA transfection reagent according to the manufacturer's instructions. The sequence of the sense strand of Nogo-B siRNA was 5'-GGAUCUCAUUGUAUGCAUATT-3', and the antisense strand sequence was 5'-UAUGACUACAAUGAGAUCCAT-3'. Adenoviral vectors were constructed carrying Nogo-B with an RFP-3 flag tag (Ad-Nogo-B) or the RFP gene alone (Ad-RFP), as described in other studies[21].

\section{KARGER}




\section{Cellular Physiology Cell Physiol Biochem 2017;41:274-285

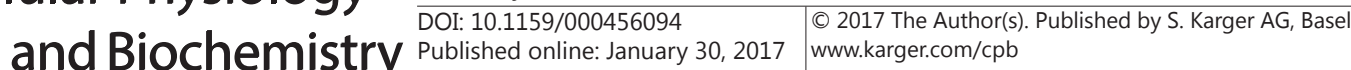 \\ Zhu et al.: Nogo-B Facilitates LPS-Mediated Immune Responses}

Reagents

LPS derived from Escherichia coli (Cat. no. L4391) was purchased from Sigma. An adenovirus of Nogo-B was purchased from Heyuan Biology Inc. SiRNA for RTN4-B (Cat. no. SI01408512) was purchased from Qiangen. The INTERFERin siRNA transfection reagent was purchased from Polyplus. Fluorescein isothiocyanate (FITC)-conjugated mouse monoclonal antibodies against MHC I, MHC II, and TLR4 were purchased from eBioscience Inc. TNF- $\alpha$, IL-1 $\beta$, MCP-1, and TGF- $\beta$ enzyme-linked immunosorbent assay (ELISA) kits were purchased from BD Bioscience.

Cell migration transwell assay

Before the experiment, cells treated with the Nogo-B adenovirus or siRNA were collected and counted, and $2 \times 10^{4}$ cells were seeded in $200 \mu \mathrm{L}$ of serum-free DMEM in the upper chamber of a 24-well transwell migration system for migration (BD Biosciences), whereas $800 \mu \mathrm{L}$ of $1 \mu \mathrm{g} / \mathrm{mL}$ LPS in DMEM with 10\% FBS was added in the lower chamber. After $24 \mathrm{~h}$ (migration), inserts were removed, and cells on the upper surface were washed with PBS and scraped with a cotton swap. Cells were fixed with $100 \%$ ethanol for $20 \mathrm{~min}$, followed by crystal violet staining. Stained inserts were cut and mounted on microscope slides. Images of migrating cells were taken using an inverted microscope (Olympus).

\section{Immunofluorescence}

Cells were incubated using primary antibodies followed by fluorophore-conjugated secondary antibodies. Detection was performed using Alexa Fluor 488 and 568 dyes, and cells were counterstained with DAPI.

\section{Flow cytometric analysis}

The expression of TLR4 and MHC I/II on the surface of RAW264.7 cells was analyzed via flow cytometry using anti-mouse antibodies. Cells were incubated with phycoerythrin-labeled antibodies against mouse TLR4 or MHC I/II (eBioscience) for $30 \mathrm{~min}$ on ice, washed, and analyzed in a FACSCalibur flow cytometer (BD Biosciences). Data was analyzed using CellQuest software (BD Biosciences) to assess the mean fluorescence intensity.

Western blot and Enzyme-linked Immunosorbent Assay

Cells were lysed in a sodium dodecyl sulfate (SDS) sample buffer by adding one third the volume of a $4 \times$ SDS sample buffer directly into the cell suspensions. The samples were then boiled for $5 \mathrm{~min}$, and $20 \mu \mathrm{g}$ was loaded onto $12 \%$ SDS-polyacrylamide gels and transferred to polyvinylidene fluoride (PVDF) membranes at $200 \mathrm{~mA}$ for $2 \mathrm{~h}$. Western blotting was performed as described in previous studies[22]. TNF- $\alpha$, MCP-1, IL-1 $\beta$, and TGF- $\beta$ concentrations in cell culture supernatants were measured using murine cytokinespecific Quantikine ELISA kits.

Statistical analysis

Data was expressed as mean $\pm \mathrm{SD}$, and statistical analysis was performed via a $t$-test or one-way analysis of variance. A $p$ value less than 0.05 was considered statistically significant.

\section{Results}

Nogo-B Expression Shows a Time- and Dose-Dependent Down-regulation by LPS in RAW264.7 Macrophages

Nogo-B has been found to be critical for monocyte-derived macrophage recruitment in cases of acute inflammation. In our previous study, we found that the up-regulation of Nogo-B increased the production of MCP-1 and alveolar macrophages in the alveolar space [22-24]. We have used macrophage cell line RAW264.7 to investigate the interaction between LPS and the expression of Nogo-B protein using a western blot in a time- and dosedependent manner during LPS stimulation and have shown that the expression of innate Nogo-B protein was significantly reduced. The reduction was proportional to the increase in LPS concentration from $1 \mathrm{ng} / \mathrm{mL}$ to $1 \mu \mathrm{g} / \mathrm{mL}$ after $12 \mathrm{~h}$ (Fig. 1A). Further reduction was 
A

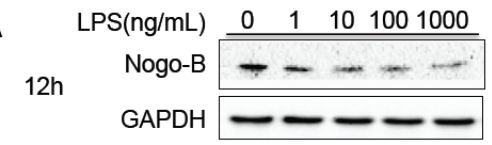

B

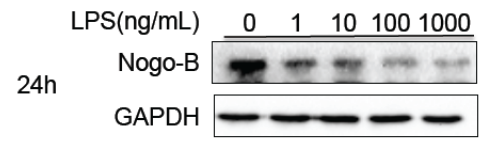

C

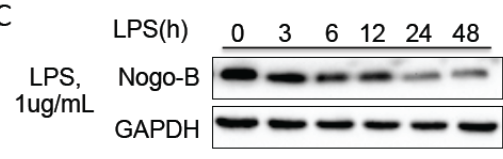

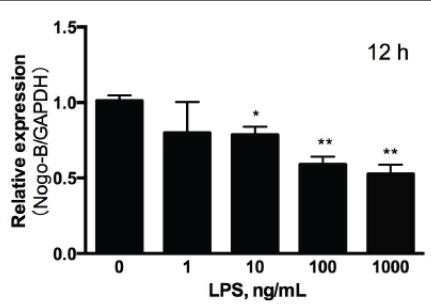
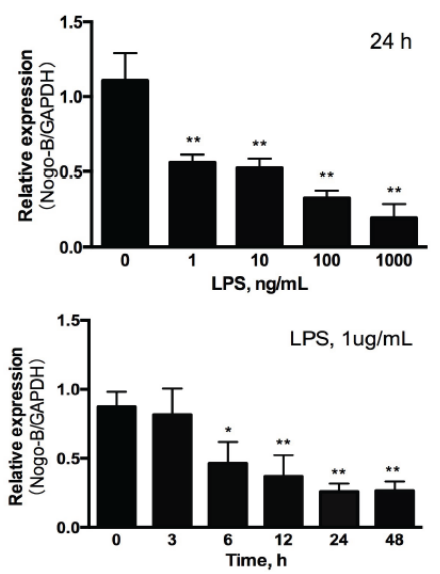

Fig. 1. Nogo-B expression in RAW264.7 cells. (A-B) The decrease in Nogo-B expression is dose-dependent in RAW264.7 after LPS treatment for $12 \mathrm{~h}$ and $24 \mathrm{~h}$, respectively. (C) The time course of Nogo-B expression declines in RAW264.7 treated with LPS $(1 \mu \mathrm{g} / \mathrm{mL}) .\left({ }^{*} \mathrm{p}<0.05,{ }^{* *} \mathrm{p}<0.01 \mathrm{vs}\right.$. control).

A

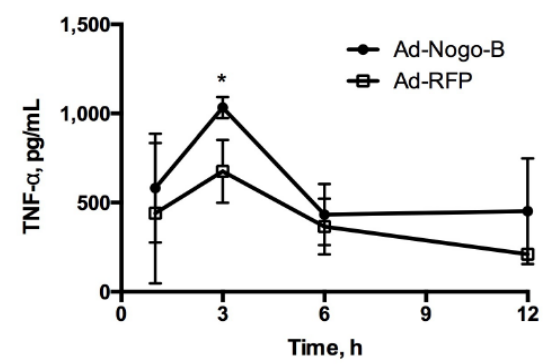

C

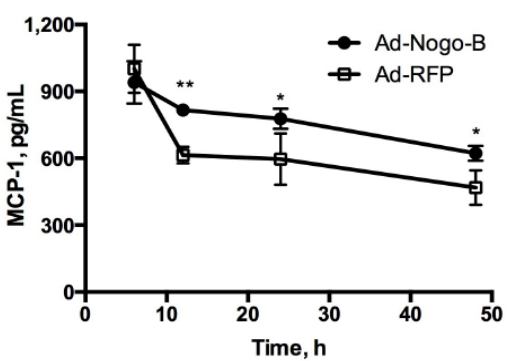

B

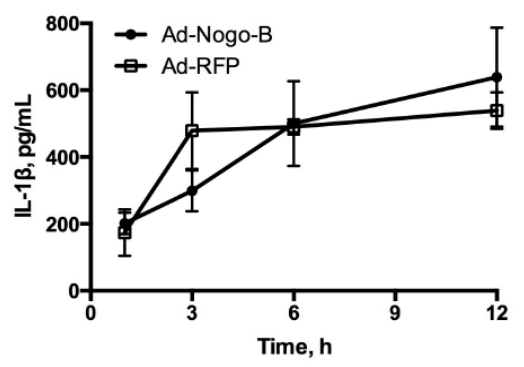

D

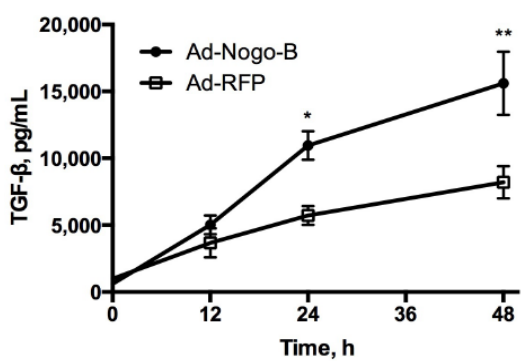

Fig. 2. Cytokines production in Nogo-B-overexpressing macrophages. (A-D) Supernatants from the cell cultures were collected, and concentrations of TNF- $\alpha$, IL-1 $\beta$, MCP-1, and TGF- $\beta$ were evaluated via ELISA. ${ }^{*} \mathrm{p}<0.05,{ }^{* *} \mathrm{p}<0.01$ compared between the Ad-RFP and Ad-Nogo-B groups, with similar results obtained in three independent experiments).

observed after $24 \mathrm{~h}$ (Fig. 1B). Accordingly, regarding time course behavior, Nogo-B levels reduced gradually after LPS administration at the dose of $1 \mu \mathrm{g} / \mathrm{mL}$ and continued to decline 
A

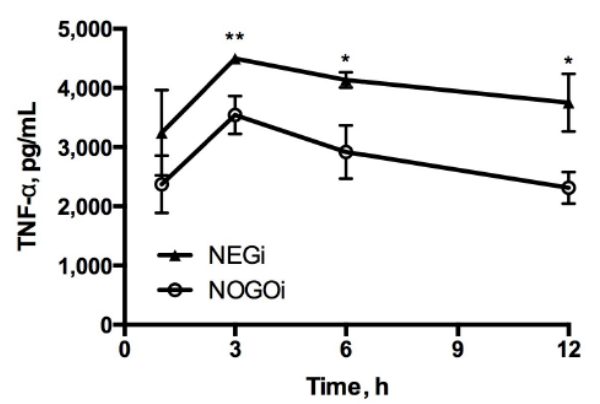

C

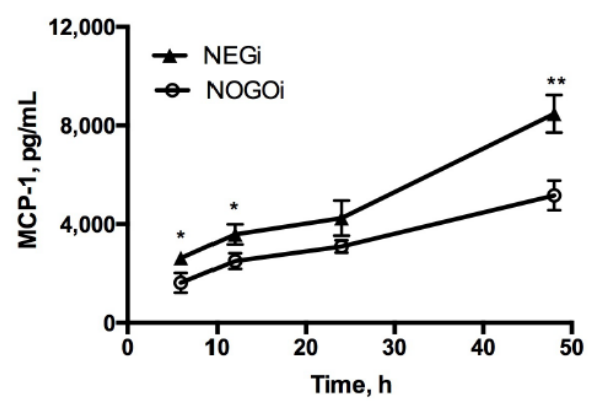

B

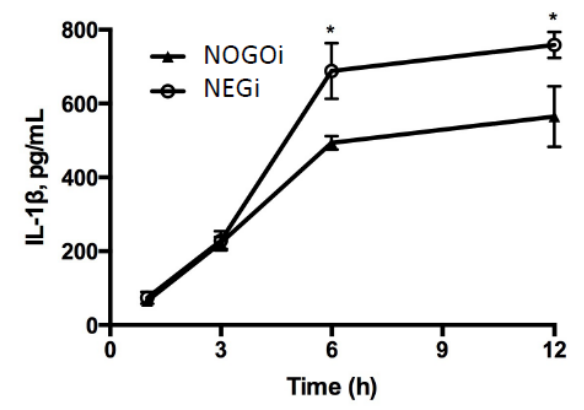

D

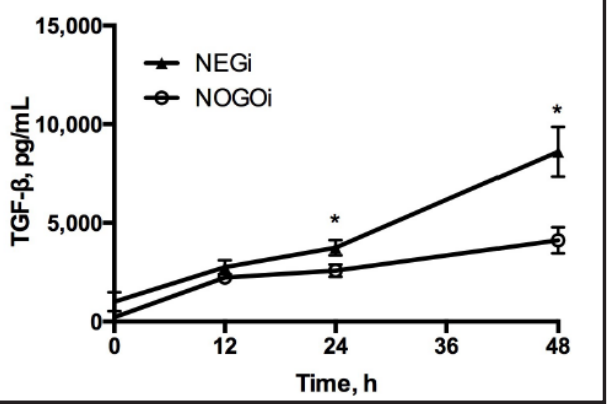

Fig. 3. Cytokine production in Nogo-B-silenced macrophages. (A-D) Supernatants from the cell cultures were obtained, and concentrations of TNF- $\alpha$, IL-1 $1 \beta$, MCP- 1 , and TGF- $\beta$ were measured via ELISA. $\left({ }^{*} p<0.05\right.$, ${ }^{* *} \mathrm{p}<0.01$ compared between the NEGi and NOGOi groups).
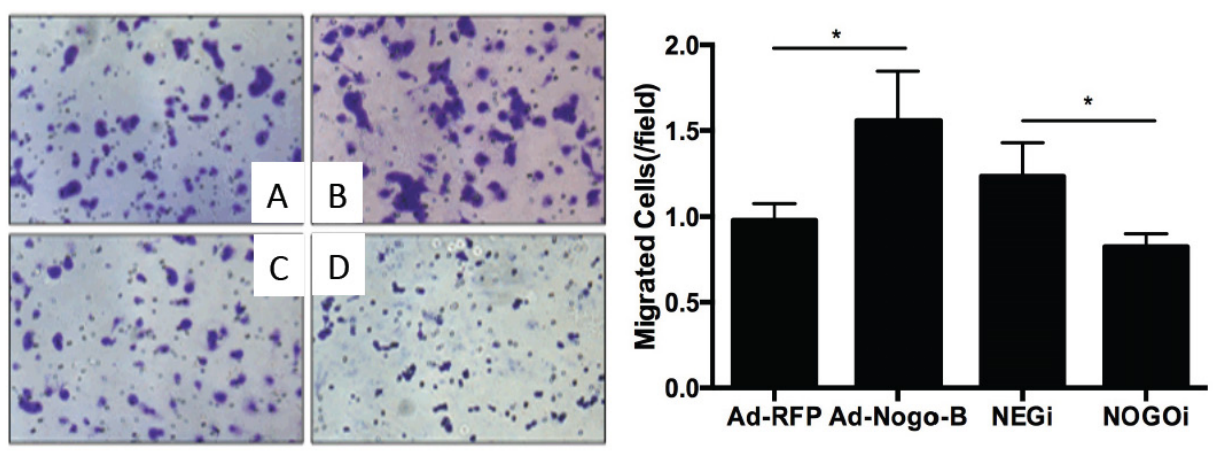

Fig. 4. Migration activities in Nogo-B-manipulated RAW264.7 cells. (A) Migration of cells in the Ad-RFP group. (B) Migration of cells in the Ad-Nogo-B group. (C) Migration of cells in the NEGi group. (D) Migration of cells in the NOGOi group. Cells were counted three times per field. $\left({ }^{*} p<0.05\right)$.

with time, with no further decrease after $24 \mathrm{~h}$ (Fig. 1C). We chose a dose of $1 \mu \mathrm{g} / \mathrm{mL}$ and a time point of $24 \mathrm{~h}$ as the standard according to which we would perform the following experiments. Taken together, these results show that macrophage RAW264.7 expresses Nogo-B endogenously and that Nogo-B levels are highly associated with LPS administration, indicating the potential role of Nogo-B in the regulation of TLR4 signaling.

Nogo-B Facilitates Cytokine production and cell migration

To further explore Nogo-B-related inflammatory response, we transfected RAW264.7 cells with an adenoviral vector carrying the Nogo-B gene with an RFP-3 flag tag, which showed a successful transfection as proven via immunofluorescence and a western blot (Fig. $7 \mathrm{~A}-\mathrm{B}$ ). We observed a fast secretion of TNF- $\alpha$ after Nogo-B overexpression at the time point 


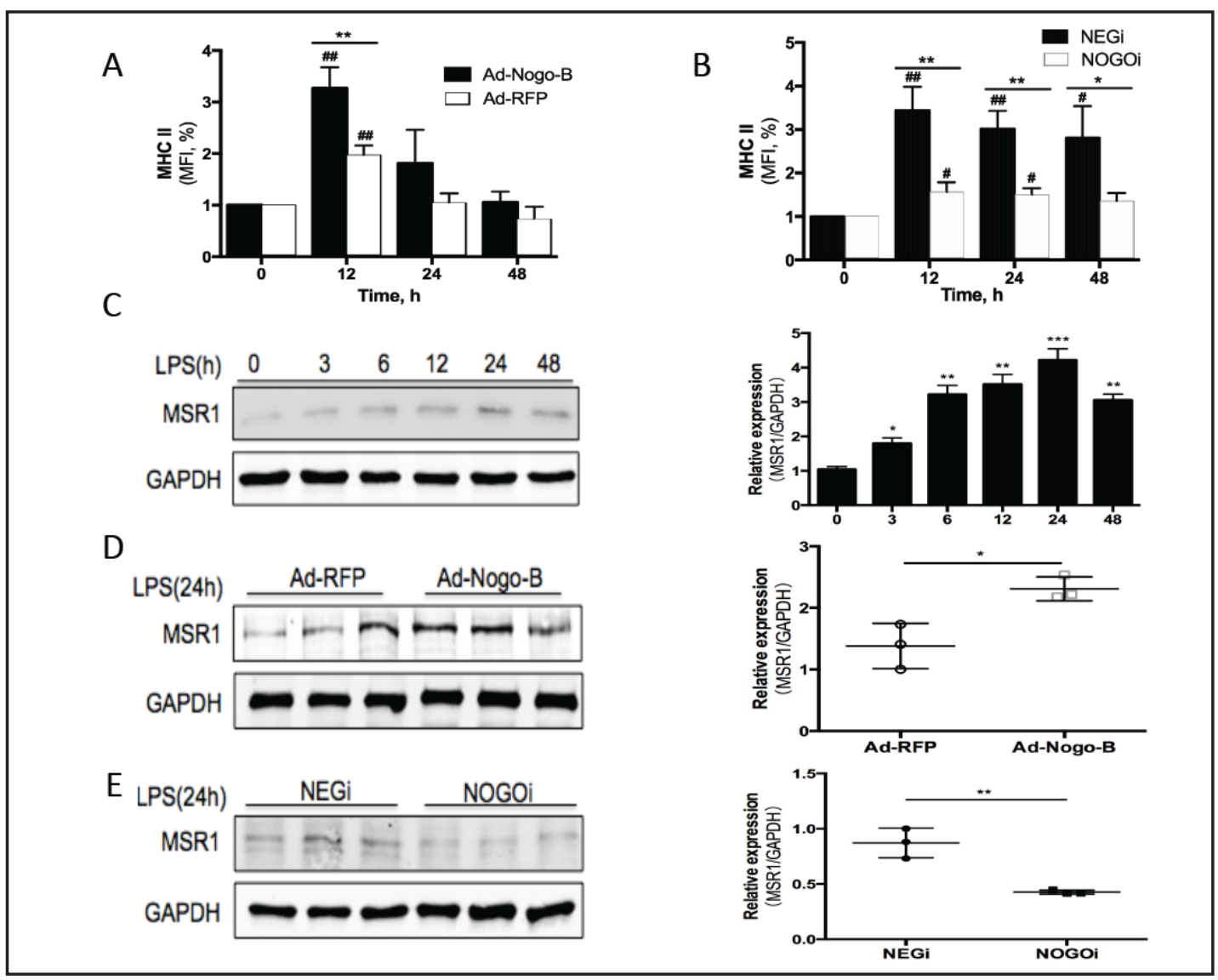

Fig. 5. Surface expression of MHC II and MSR1 on RAW264.7 cells. (A) Plotting of relative MHC II surface mean fluorescence tested via flow cytometry after LPS treatment for the indicated time periods (mean fluorescence intensity at time 0 as 100\%). Mean fluorescence intensity of MHC II in Nogo-B-overexpressing macrophages. (B) Mean fluorescence intensity of MHC II in Nogo-B-silenced RAW264.7 cells. (C) MSR1 expression after treatment with LPS. (D) MSR1 expression increased after overexpression of Nogo-B with LPS exposure. (E) MSR1 expression declined after the down-regulation of Nogo-B during LPS treatment. $\left({ }^{*} \mathrm{p}<0.05,{ }^{* *} \mathrm{p}<0.01 ;{ }^{*} \mathrm{p}<0.05\right.$ compared between the Ad-RFP and Ad-Nogo-B groups or the NEGi and NOGOi groups, ${ }^{\# \#} \mathrm{p}<0.01$ compared with time 0 in each group; all values are presented as the mean \pm SD of three independent experiments).

of $3 \mathrm{~h}$ after administration of LPS at $1 \mu \mathrm{g} / \mathrm{mL}$, which recovered to the baseline after $6 \mathrm{~h}$, with no great difference in cytokine IL-1 $\beta$ levels (Fig. 2A-B). The production of MCP-1 showed a descending trend after LPS exposure, although the overexpression group was still higher than the control cells (Fig. 2C). The expression of TGF- $\beta$ notably increased after $24 \mathrm{~h}$ and continued to increase until $48 \mathrm{~h}$ compared to the control group (Fig. 2D).

We silenced the expression of Nogo-B using a stable transfection vector with Nogo-B siRNA to further evaluate the inflammatory cytokines in Nogo-B-null cells. Nogo-B expression was significantly suppressed by Nogo-B siRNA but not by corresponding scrambled controls (Fig. 6A). We observed a decreased secretion of cytokine TNF- $\alpha$ in both groups $3 \mathrm{~h}$ after treatment with $1 \mu \mathrm{g} / \mathrm{mL}$ LPS. However, compared to the control group, TNF- $\alpha$ was more depressed in Nogo-B-deficient cells (Fig. 3A). Additionally, the productions of IL-1 $\beta$, MCP1 , and TGF- $\beta$ in the NOGOi group were all inhibited after LPS treatment even though each cytokine was stimulated with LPS (Fig. 3B-D).

We next examined cell migration ability via a transwell assay. Our results suggest that Nogo-B transfection enhances the migration ability of RAW264.7 cells by a factor of 1.8 after $24 \mathrm{~h}$ (Fig. 4A-B). However, the number of macrophage cells was over 30\% lower in

\section{KARGER}




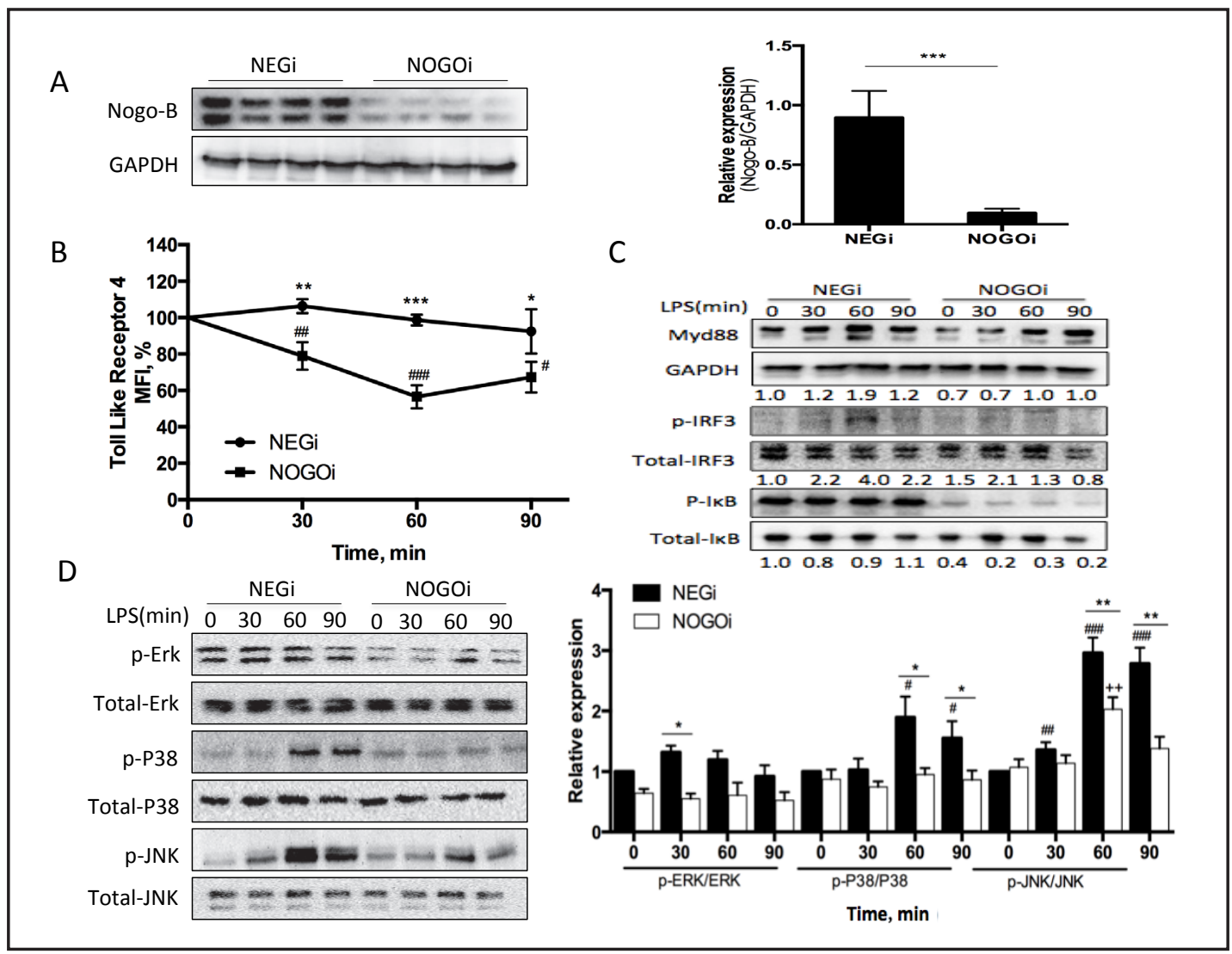

Fig. 6. Nogo-B-decreased TLR4 and inhibited MAPK pathway. (A) The down-regulation of Nogo-B expression in RAW264.7. Protein levels were determined via Image J analysis. (B) The mean fluorescence intensity of TLR4 was shown between the NEGi and NOGOi groups after LPS treatment. (C) The expression of MYD88, p-IRF3, and p-IкB was detected via a western blot. The given numbers were measured via densitometry. (D) Phosphorylated proteins of ERK, p38, and JNK were revealed via a western blot. The protein levels were determined via Image J analysis. $\left({ }^{*} \mathrm{p}<0.05,{ }^{* *} \mathrm{p}<0.01\right.$ compared between the NEGi and NOGOi groups; ${ }^{\#} \mathrm{p}<0.05$, ${ }^{\# \#} \mathrm{p}<0.01$ compared with time 0 in the NOGOi group).

the Nogo-B siRNA group than in the negative control (Fig. 4C-D). These results support that Nogo-B is required for the full promotion of microphage activities.

\section{Nogo-B enhances the Antigen-Presenting and Scavenging Capacities of Macrophages}

To establish the effects of Nogo-B on the antigen-presenting capacity of macrophages and on MHC I and II expression, a western blot and flow cytometry were conducted after LPS exposure using the aforementioned constructs overexpressing or silencing Nogo-B. The physiological function of MHC I mainly mediates the presentation of endogenous antigens, whereas MHC II presents exogenous antigens. MHC II expression increased significantly along time after overexpression of Nogo-B, which peaked at $12 \mathrm{~h}$ and then dropped as time lapsed (Fig. 5A). As presented in Fig. 5B, we observed a similar trend of more suppression in the absence of Nogo-B. However, there was no significant difference in MHC I expression between the two groups (data not shown).

To further determine the effects of Nogo-B on the phagocytic capacity of macrophages, we measured the expression of MSR1 after LPS administration. As shown in Fig. 5C, MSR1 expression peaked at $24 \mathrm{~h}$ and then began to decrease. Next, we overexpressed Nogo-B and observed that MSR1 expression was enhanced after LPS exposure at $24 \mathrm{~h}$ (Fig. 5D). Accordingly, this protein declined with a lack of Nogo-B (Fig. 5E). These findings show that 


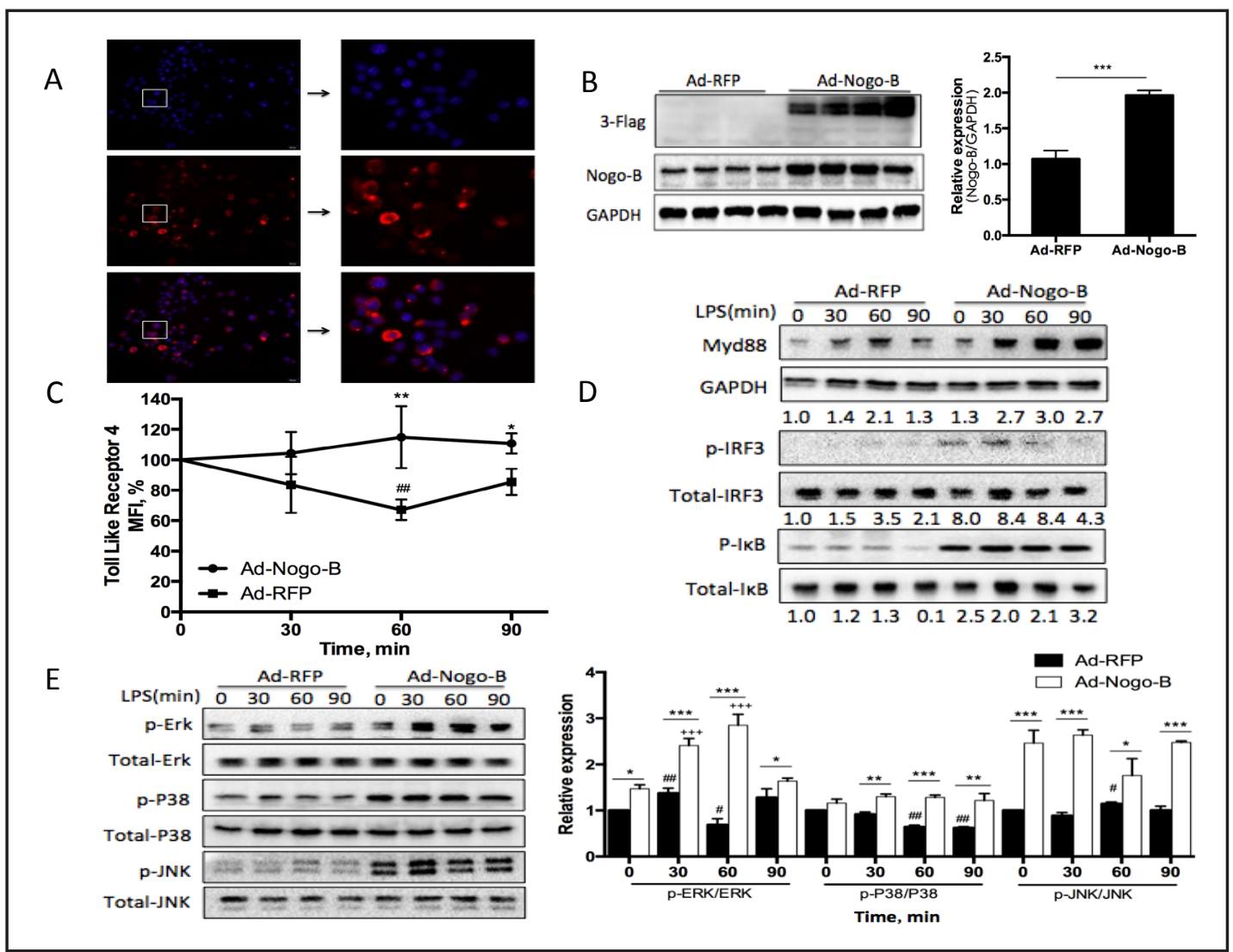

Fig. 7. Nogo-B-enhanced TLR4 and activated MAPK pathway. (A) The blue spots show the positions of the nuclei of macrophages. The red fluorescence indicates the adenovirus carrying the Nogo-B genes. The merged images present the successful transfection of Nogo-B into the macrophage cytoplasm. White boxes indicate the general location of the higher-magnification image shown on the right side. (B) The up-regulation of Nogo-B expression in RAW264.7. Protein levels were evaluated via Image J analysis. (C) The mean fluorescence intensity of TLR4 was demonstrated between Ad-RFP and Ad-Nogo-B after LPS treatment. (D) The expression of MYD88, p-IRF3, and p-IкB was analyzed via a western blot. Given numbers were determined via densitometry. (E) Phosphorylated proteins of ERK, p38, and JNK were shown via a western blot. Protein levels were measured via Image J analysis. ( ${ }^{*} \mathrm{p}<0.05,{ }^{* *} \mathrm{p}<0.01$ compared between the Ad-RFP and Ad-Nogo-B groups; ${ }^{\#} \mathrm{p}<0.05,{ }^{\# \#} \mathrm{p}<0.01$ compared with time 0 in each group; data represents three independent experiments).

Nogo-B exerts a positive effect on antigen presentation and might influence phagocytic capacity in RAW264.7.

Nogo-B Affects Cell-Surface Expression of TLR4 and Regulates MAPK Pathway Activation

Mechanistically, we attempted to determine whether Nogo-B affects TLR4 expression and further regulates the functional integrity of intracellular signal pathway MAPKs. We monitored changes to TLR4 on the surface of RAW264.7 after knocking down the Nogo-B and discovered that the mean fluorescence intensity of TLR4 in the NOGOi group decreased in $30 \mathrm{~min}$, reaching a minimum at $60 \mathrm{~min}$ and remaining lower than the baseline $90 \mathrm{~min}$ after LPS simulation (Fig. 6B). We then analyzed the subsequent phosphorylation proteins activated by either MYD88 or non-MYD88 (TIR-domain-containing adapter-inducing interferon- $\beta$ )-dependent pathways to establish how Nogo-B may affect TLR4-mediated downstream signaling. The activation of extracellular-regulated kinase 1 and 2 (ERK1 and ERK2) was altered to a lesser extent after Nogo-B knockdown compared to the control siRNAtransfected RAW264.7 cells by LPS stimulation (Fig. 6D). Similarly, the phosphorylated forms 
of c-Jun N-terminal kinase (JNK) and p38 were also inhibited, usually peaking at $30 \mathrm{~min}$. Analysis of LPS-induced MYD88 and p-IRF3 was demonstrated in the same responses (Fig. 6C). Additionally, we observed the impaired degradation of phosphorylated I- $\kappa B$, which further inhibited the activation of NF- $\mathrm{KB}$, consistent with the decreased secretion of cytokines.

To further ascertain how Nogo-B mediates the regulation of TLR4 pathways in macrophages, we overexpressed the Nogo-B protein, which increased TLR4 surface expression and prevented TLR4 down-regulatory effects from LPS exposure within 60 min (Fig. 7C). The phosphorylation levels of ERK1/2, JNK, and p38 were significantly enhanced, and the expression of MYD88, p-IRF3, and p-IкB was activated, consistent with the consequences of an enhancement of inflammatory cytokines (Fig. 7D-E). Taken together, this data indicates that Nogo-B is essential for optimal surface expression of TLR4, which further modifies LPS-initiated activation of the MAPK pathway, eventually supporting the notion that Nogo-B regulates TLR4 signaling pathways in the presence of LPS.

\section{Discussion}

Nogo-B is one of the three main isoforms of RTN-4 who belongs to the big family of Reticulons which share a common C-terminal domain. The other two members of RTN4 are Nogo-A and - C. In these three proteins, Nogo-A protein is more known as a potent neurite outgrowth inhibitor of high molecular mass mainly expressed in white-matter oligodendrocytes and myelin, playing a variety of roles in neurodegenerative diseases [25]. Therefore, Nogo-A presents close association with neurodegenerative diseases more than Nogo-B. In addition, as a splice variant of Nogo-A, Nogo-B is highly expressed in immune cells in the lung, especially in macrophages and more involved in the regulation of apoptosis, remodeling of vasculature and tissue repair as well as the inflammation process. Thus, we focus this study on revealing the mechanism of Nogo-B in responding acute inflammation in Lung disease. We have examined the effects of Nogo-B expression on the regulation of inflammatory responses in mouse macrophage cell line RAW264.7 after LPS exposure. We have shown that Nogo-B positively regulates TLR4 expression and initiates the release of cytokines and chemokines during LPS treatment. In addition, we have demonstrated a strong correlation between the Nogo-B protein and cell migration, which suggests that Nogo-B is essential for the recruitment of microphages during LPS administration. Furthermore, Nogo-B expression regulates MHC II cell-surface expression in macrophages, promoting an antigenpresenting ability and activating the acquired immune system. Therefore, loss of Nogo-B in macrophages may cause a defect in immune responses. However, the exact mechanisms underlying the ability of Nogo-B to regulate macrophages are yet to be understood.

We have found that Nogo-B enhances the secretion of cytokines such as TNF- $\alpha$, IL$1 \beta$, and MCP-1, which is consistent with the findings of Di Lorenzo et al. that the loss of Nogo-B in macrophages reduces innate immune response [26]. Studies have shown that inhibiting MCP-1 causes capillary-alveolar barrier damage and reduces the recruitment of macrophages [27-29]. Given the important and protective role of MCP-1 in inflammatory diseases, this may explain why the overexpression of Nogo-B enhanced the migration abilities of RAW264.7 cells in our study. We have also reported a gradual increase in TGF- $\beta$ expression concomitant with a decrease in TNF- $\alpha$. This shift from pro-inflammatory to antiinflammatory cytokine expression may signal the start of tissue repair and regeneration. Likewise, the high expression of MSR1 could indicate that Nogo-B might intensify the competence of the phagocytosis of invading bacteria or apoptotic inflammatory cells, inducing an anti-inflammatory phenotype of macrophages (M2 phenotype, alternatively activated macrophages)[6, 30,31]. However, the surface expression of MSR1 is an indirect tool to test phagocytic behavior. Further experiments must be conducted.

In addition, macrophages are involved in triggering the response of the adaptive immune system by processing and presenting antigens [5]. Nogo-B deficiency is associated 
with decreased MHC II expression, indicating a diminished capacity to scavenge and present exotic antigens. MHC II, designed to firmly bind with and present fragments of exogenous antigens to $\mathrm{T}$ cells, is primarily expressed on antigen-presenting cell surfaces, including macrophages, B cells, and DCs [32]. In the present study, Nogo-B up-regulated MHC II expression, demonstrating that Nogo-B might promote the antigen-presenting ability of macrophages to exogenous antigens. However, the expression of MHC I, which mainly mediates the presentation of endogenous antigens, was not much different. These findings suggest that Nogo-B is involved in initiating innate responses as well as adaptive immune responses via the processing and presentation of antigens.

TLRs are abundantly expressed in macrophages and play an important role in Gramnegative sepsis or artificial LPS exposure to trigger the innate immune system [33, 34]. Specifically, TLR4 is essential for the opsonization of LPS, a physiologic membrane-bound component of the outer membrane of Gram-negative bacteria. The regulation of TLR function has been the subject of intensive research. Studies have suggested that some RTN family members could play roles in protein transport from the ER to other membrane compartments, including the cell membrane [35]. For example, RTN2B could enhance the trafficking of glutamate transporter EAAC1 from the ER to the cell surface. In addition, RTN2 is also involved in the ER morphogenesis which may cause axon-degenerative disorder such as hereditary spastic paraplegias in the mutation or abnormal status of this protein[25]. Recently, Nogo-B has also been identified as a positive molecule modifying the trafficking of nucleic acid-sensing TLRs [36].

In the present study, we have found that Nogo-B can increase the surface expression of TLR4 and activate the down-stream MAPK signaling pathway. However, whether Nogo-B regulates TLR4 signaling in macrophages, such as with nucleic acid-sensing TLRs, by replenishing TLR4 from the ER to the plasma membrane remains to be explored [37]. The MAPKs are a family of serine/threonine kinases consisting of extracellular-regulated kinase, JNK/stress-activated protein kinase (SAPK), and p38 MAPK, which is fundamentally associated with numerous changes in cell function, including cytokine expression, proliferation, and apoptosis [38-40]. Recent studies have discovered that Nogo-B can undergo Ser phosphorylation in vitro through the SAPK2/p38 pathway, which suggests Nogo-B may be involved in the biological roles of MAPK pathways [41]. In our study, Nogo-B activated the phosphorylation of MAP kinases (p38, JNK, and ERK) simultaneously. At the upstream of NF- $\kappa B$, we checked the degradation of phosphorylated I- $\kappa B$, which was positively influenced by Nogo-B and may further cause NF- $\mathrm{KB}$ transcriptional activities, while more specific mechanisms remain unknown.

In conclusion, our data demonstrates the function and possible mechanism of Nogo-B in modulating macrophages' mediated immune responses through TLR4/MAPK signaling pathways. Further studies are required to detail the molecular mechanisms of the trafficking of TLR4s in the regulation of Nogo-B.

\section{Acknowledgement}

This study was supported by grants from National Natural Science Foundation of China (Grant No.81200030, No. 81470265 and No. 81302580).

\section{Disclosure Statement}

The authors have declared that no competing interests exist.

\section{References}

1 Beutler B, Jiang Z, Georgel P, Crozat K, Croker B, Rutschmann S, Du X, Hoebe K: Genetic analysis of host resistance: Toll-like receptor signaling and immunity at large. Annu Rev Immunol 2006;24:353-389. 


\section{Cellular Physiology Cell Physiol Biochem 2017;41:274-285 \begin{tabular}{c|c|c|} 
DOI: 10.1159/000456094 & C 2017 The Author(s). Published by S. Karger AG, Basel \\
www.karger.com/cpb
\end{tabular}

2 Barton GM, Kagan JC: A cell biological view of Toll-like receptor function: regulation through compartmentalization. Nat Rev Immunol 2009;9:535-542.

3 Kawai T, Akira S: The role of pattern-recognition receptors in innate immunity: update on Toll-like receptors. Nat Immunol 2010;11:373-384.

4 Lakhani SA, Bogue CW: Toll-like receptor signaling in sepsis. Curr Opin Pediatr 2003;15:278-282.

5 Gou Z, Jiang S, Zheng C, Tian Z, Lin X: Equol Inhibits LPS-Induced Oxidative Stress and Enhances the Immune Response in Chicken HD11 Macrophages. Cell Physiol Biochem 2015;36:611-621.

-6 Zhou Y, Zhang T, Wang X, Wei X, Chen Y, Guo L, Zhang J, Wang C: Curcumin Modulates Macrophage Polarization Through the Inhibition of the Toll-Like Receptor 4 Expression and its Signaling Pathways. Cell Physiol Biochem 2015;36:631-641. Kang JY, Lee JO: Structural biology of the Toll-like receptor family. Annu Rev Biochem 2011;80:917-941. Meng Y, Chen C, Tian C, Du J, Li HH: Angiotensin II-induced Egr-1 expression is suppressed by peroxisome proliferator-activated receptor-gamma ligand 15d-PGJ(2) in macrophages. Cell Physiol Biochem 2015;35:689-698.

-9 Watari A, Yutsudo M: Multi-functional gene ASY/Nogo/RTN-X/RTN4: apoptosis, tumor suppression, and inhibition of neuronal regeneration. Apoptosis 2003;8:5-9.

10 Oertle T, Huber C, van der Putten H, Schwab ME: Genomic structure and functional characterisation of the promoters of human and mouse nogo/rtn4. J Mol Biol 2003;325:299-323.

11 Chiurchiu V, Maccarrone M, Orlacchio A: The role of reticulons in neurodegenerative diseases. Neuromolecular Med 2014;16:3-15.

12 Schwab ME: Functions of Nogo proteins and their receptors in the nervous system. Nat Rev Neurosci 2010;11:799-811.

13 Drozdz K, Grzegorek I, Chmielewska M, Gomulkiewicz A, Jablonska K, Piotrowska A, Karczewski M, Janczak D, Patrzalek D, Dziegiel P, Szuba A: Nogo-B expression, in arterial intima, is impeded in the early stages of atherosclerosis in humans. APMIS 2014;122:742-749.

- 14 Li Q Qi B, Oka K, Shimakage M, Yoshioka N, Inoue H, Hakura A, Kodama K, Stanbridge EJ, Yutsudo M: Link of a new type of apoptosis-inducing gene ASY/Nogo-B to human cancer. Oncogene 2001;20:3929-3936.

15 Oertle T, Merkler D, Schwab ME: Do cancer cells die because of Nogo-B? Oncogene 2003;22:1390-1399.

-16 Acevedo L, Yu J, Erdjument-Bromage H, Miao RQ, Kim JE, Fulton D, Tempst P, Strittmatter SM, Sessa WC: A new role for Nogo as a regulator of vascular remodeling. Nat Med 2004;10:382-388.

17 Schanda K, Hermann M, Stefanova N, Gredler V, Bandtlow C, Reindl M: Nogo-B is associated with cytoskeletal structures in human monocyte-derived macrophages. BMC Res Notes 2011;4:6.

18 Yu J, Fernandez-Hernando C, Suarez Y, Schleicher M, Hao Z, Wright PL, DiLorenzo A, Kyriakides TR, Sessa WC: Reticulon 4B (Nogo-B) is necessary for macrophage infiltration and tissue repair. Proc Natl Acad Sci U S A 2009;106:17511-17516.

19 Marin EP, Moeckel G, Al-Lamki R, Bradley J, Yan Q Wang T, Wright PL, Yu J, Sessa WC: Identification and regulation of reticulon 4B (Nogo-B) in renal tubular epithelial cells. Am J Pathol 2010;177:2765-2773.

20 Kondo Y, Jadlowiec CC, Muto A, Yi T, Protack C, Collins MJ, Tellides G, Sessa WC, Dardik A: The Nogo-B-PirB axis controls macrophage-mediated vascular remodeling. PLoS One 2013;8:e81019.

-21 Ng P, Parks RJ, Cummings DT, Evelegh CM, Graham FL: An enhanced system for construction of adenoviral vectors by the two-plasmid rescue method. Hum Gene Ther 2000;11:693-699.

22 Xu W, Zhu Y, Ning Y, Dong Y, Huang H, Zhang W, Sun Q Li Q: Nogo-B protects mice against lipopolysaccharide-induced acute lung injury. Sci Rep 2015;5:12061.

23 Zhang D, Utsumi T, Huang HC, Gao L, Sangwung P, Chung C, Shibao K, Okamoto K, Yamaguchi K, Groszmann RJ, Jozsef L, Hao Z, Sessa WC, Iwakiri Y: Reticulon 4B (Nogo-B) is a novel regulator of hepatic fibrosis. Hepatology 2011;53:1306-1315.

24 Beck-Schimmer B, Schwendener R, Pasch T, Reyes L, Booy C, Schimmer RC: Alveolar macrophages regulate neutrophil recruitment in endotoxin-induced lung injury. Respir Res 2005;6:61.

-25 Montenegro G, Rebelo AP, Connell J, Allison R, Babalini C, D'Aloia M, Montieri P, Schule R, Ishiura H, Price J, Strickland A, Gonzalez MA, Baumbach-Reardon L, Deconinck T, Huang J, Bernardi G, Vance JM, Rogers MT, Tsuji S, De Jonghe P, Pericak-Vance MA, Schols L, Orlacchio A, Reid E, Zuchner S: Mutations in the ERshaping protein reticulon 2 cause the axon-degenerative disorder hereditary spastic paraplegia type 12 . J Clin Invest 2012;122:538-544. 


\section{Cellular Physiology Cell Physiol Biochem 2017;41:274-285 \begin{tabular}{l|l} 
DOI: 10.1159/000456094 30, 2017 & $\begin{array}{l}\text { O 2017 The Author(s). Published by S. Karger AG, Basel } \\
\text { www.karger.com/cpb }\end{array}$ \\
\hline
\end{tabular} \\ Zhu et al.: Nogo-B Facilitates LPS-Mediated Immune Responses}

-26 Di Lorenzo A, Manes TD, Davalos A, Wright PL, Sessa WC: Endothelial reticulon-4B (Nogo-B) regulates ICAM-1-mediated leukocyte transmigration and acute inflammation. Blood 2011;117:2284-2295.

27 Maus UA, Srivastava M, Paton JC, Mack M, Everhart MB, Blackwell TS, Christman JW, Schlondorff D, Seeger W, Lohmeyer J: Pneumolysin-induced lung injury is independent of leukocyte trafficking into the alveolar space. J Immunol 2004;173:1307-1312.

28 Suresh MV, Yu B, Machado-Aranda D, Bender MD, Ochoa-Frongia L, Helinski JD, Davidson BA, Knight PR, Hogaboam CM, Moore BB, Raghavendran K: Role of macrophage chemoattractant protein-1 in acute inflammation after lung contusion. Am J Respir Cell Mol Biol 2012;46:797-806.

-29 Kanikarla-Marie P, Jain SK: Role of Hyperketonemia in Inducing Oxidative Stress and Cellular Damage in Cultured Hepatocytes and Type 1 Diabetic Rat Liver. Cell Physiol Biochem 2015;37:2160-2170.

-30 Matute-Bello G, Martin TR: Science review: apoptosis in acute lung injury. Crit Care 2003;7:355-358.

-31 Gordon S, Martinez FO: Alternative activation of macrophages: mechanism and functions. Immunity 2010;32:593-604.

32 Ge J, Wang Y, Feng Y, Liu H, Cui X, Chen F, Tai G, Liu Z: Direct effects of activin A on the activation of mouse macrophage RAW264.7 cells. Cell Mol Immunol 2009;6:129-133.

33 Xiang M, Fan J: Pattern recognition receptor-dependent mechanisms of acute lung injury. Mol Med 2010;16:69-82.

34 Qian C, Cao X: Regulation of Toll-like receptor signaling pathways in innate immune responses. Ann N Y Acad Sci 2013;1283:67-74.

-35 Liu Y, Vidensky S, Ruggiero AM, Maier S, Sitte HH, Rothstein JD: Reticulon RTN2B regulates trafficking and function of neuronal glutamate transporter EAAC1. J Biol Chem 2008;283:6561-6571.

- 36 Kimura T, Endo S, Inui M, Saitoh SI, Miyake K, Takai T: Endoplasmic Protein Nogo-B (RTN4-B) Interacts with GRAMD4 and Regulates TLR9-Mediated Innate Immune Responses. J Immunol 2015;10.4049/ jimmunol.1402006

37 Pula B, Werynska B, Olbromski M, Muszczynska-Bernhard B, Chabowski M, Janczak D, Zabel M, PodhorskaOkolow M, Dziegiel P: Expression of Nogo isoforms and Nogo-B receptor (NgBR) in non-small cell lung carcinomas. Anticancer Res 2014;34:4059-4068.

-38 Tang H, Sun Y, Shi Z, Huang H, Fang Z, Chen J, Xiu Q, Li B: YKL-40 induces IL-8 expression from bronchial epithelium via MAPK (JNK and ERK) and NF-kappaB pathways, causing bronchial smooth muscle proliferation and migration. J Immunol 2013;190:438-446.

-39 Shen YH, Godlewski J, Zhu J, Sathyanarayana P, Leaner V, Birrer MJ, Rana A, Tzivion G: Cross-talk between JNK/SAPK and ERK/MAPK pathways: sustained activation of JNK blocks ERK activation by mitogenic factors. J Biol Chem 2003;278:26715-26721.

-40 Xue J, Chen F, Wang J, Wu S, Zheng M, Zhu H, Liu Y, He J, Chen Z: Emodin protects against concanavalin A-induced hepatitis in mice through inhibiting activation of the p38 MAPK-NF-kappaB signaling pathway. Cell Physiol Biochem 2015;35:1557-1570.

-41 Rousseau S, Peggie M, Campbell DG, Nebreda AR, Cohen P: Nogo-B is a new physiological substrate for MAPKAP-K2. Biochem J 2005;391:433-440. 